\title{
Front Matter: Volume 11240
}

, "Front Matter: Volume 11240," Proc. SPIE 11240, Photons Plus Ultrasound: Imaging and Sensing 2020, 1124001 (16 March 2020); doi: $10.1117 / 12.2566009$

SPIE. Event: SPIE BiOS, 2020, San Francisco, California, United States 


\title{
PROGRESS IN BIOMEDICAL OPTICS AND IMAGING
}

\section{Photons Plus Ultrasound: Imaging and Sensing 2020}

\author{
Alexander A. Oraevsky \\ Lihong V. Wang \\ Editors
}

2-5 February 2020

San Francisco, California, United States

Sponsored by

SPIE

Cosponsored by

SENO Medical Instruments, Inc. (United States)

Published by

SPIE 
The papers in this volume were part of the technical conference cited on the cover and title page. Papers were selected and subject to review by the editors and conference program committee. Some conference presentations may not be available for publication. Additional papers and presentation recordings may be available online in the SPIE Digital Library at SPIEDigitalLibrary.org.

The papers reflect the work and thoughts of the authors and are published herein as submitted. The publisher is not responsible for the validity of the information or for any outcomes resulting from reliance thereon.

Please use the following format to cite material from these proceedings:

Author(s), "Title of Paper," in Photons Plus Ultrasound: Imaging and Sensing 2020, edited by Alexander A. Oraevsky, Lihong V. Wang, Proceedings of SPIE Vol. 11240 (SPIE, Bellingham, WA, 2020) Seven-digit Article CID Number.

ISSN: 1605-7422

ISSN: 2410-9045 (electronic)

ISBN: 9781510632431

ISBN: 9781510632448 (electronic)

Published by

SPIE

P.O. Box 10, Bellingham, Washington 98227-0010 USA

Telephone +1 3606763290 (Pacific Time) · Fax +1 3606471445

SPIE.org

Copyright (c) 2020, Society of Photo-Optical Instrumentation Engineers.

Copying of material in this book for internal or personal use, or for the internal or personal use of specific clients, beyond the fair use provisions granted by the U.S. Copyright Law is authorized by SPIE subject to payment of copying fees. The Transactional Reporting Service base fee for this volume is $\$ 21.00$ per article (or portion thereof), which should be paid directly to the Copyright Clearance Center (CCC), 222 Rosewood Drive, Danvers, MA 01923. Payment may also be made electronically through CCC Online at copyright.com. Other copying for republication, resale, advertising or promotion, or any form of systematic or multiple reproduction of any material in this book is prohibited except with permission in writing from the publisher. The CCC fee code is 1605$7422 / 20 / \$ 21.00$.

Printed in the United States of America by Curran Associates, Inc., under license from SPIE.

Publication of record for individual papers is online in the SPIE Digital Library.

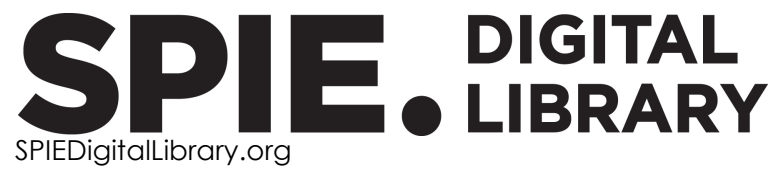

Paper Numbering: Proceedings of SPIE follow an e-First publication model. A unique citation identifier (CID) number is assigned to each article at the time of publication. Utilization of CIDs allows articles to be fully citable as soon as they are published online, and connects the same identifier to all online and print versions of the publication. SPIE uses a seven-digit CID article numbering system structured as follows:

- The first five digits correspond to the SPIE volume number.

- The last two digits indicate publication order within the volume using a Base 36 numbering system employing both numerals and letters. These two-number sets start with 00, 01, 02, 03, 04, $05,06,07,08,09,0 A, 0 B \ldots$ OZ, followed by 10-1Z, 20-2Z, etc. The CID Number appears on each page of the manuscript. 


\title{
Contents
}

\author{
ix Authors \\ xi Conference Committee \\ xV Introduction
}

CLINICAL IMAGING I: IN VIVO

$1124004 \quad$ Multispectral photoacoustic assessment of thyroid cancer nodules in vivo [1 1240-2]

1124006 Wide-field multispectral photoacoustic imaging of human melanomas in vivo [11240-4]

CLINICAL IMAGING II: EX VIVO

$112400 \mathrm{~A} \quad$ Assessment of human colorectal cancer using co-registered photoacoustic and ultrasound tomography system [11240-8]

11240 OC Study for evaluation of skin aging with photoacoustic microscopy [1 1240-10]

11240 OD Monte Carlo simulation for improving spectral photoacoustic imaging-based oxygen saturation estimation of human placental tissue [1 1240-11]

TOWARDS CLINICAL IMAGING

11240 OJ Deep learning-based speed of sound aberration correction in photoacoustic images [1 1240-17]

11240 OK Three-dimensional photoacoustic/ultrasound handheld scanner for clinical translation [1 1240-18]

$11240 \mathrm{OL} \quad$ A preclinical small animal imaging plafform combining multi-angle photoacoustic and fluorescence projections into co-registered 3D maps [1 1240-130]

\section{SMALL-ANIMAL IMAGING}

11240 OM LED-based photoacoustic imaging for early detection of joint inflammation in rodents: towards achieving $3 R$ s in rheumatoid arthritis research [11240-20]

11240 OR Imaging tumor vasculature using LED based photoacoustic system [1 1240-26] 
OPTICAL SENSING AND GENERATION OF US I

11240 OS SNR-enhanced fiber-laser ultrasound sensors for photoacoustic tomography* [11240-27]

OPTICAL SENSING AND GENERATION OF US II

$1124010 \quad$ Ultra-high sensitive all-optical photoacoustic transducers [11240-35]

CONTRAST AGENTS

1124017 Visualization of microparticle flow in the mouse brain in an intracardiac perfusion model [1 1240-42]

1124018 Calcium-sensitive photoacoustic probe for noninvasive extracellular calcium monitoring [1 1240-43]

\section{MULTI-MODALITY IMAGING}

$112401 \mathrm{P} \quad$ Seamlessly integrated optical and acoustical imaging systems through transparent ultrasonic transducer [1 1240-62]

$112401 Q \quad$ Photothermal strain imaging for diagnosis of non-alcoholic fatty liver disease [11240-63]

$112401 \mathrm{IT} \quad$ LED-based photoacoustic imaging system: why it achieves the same signal to noise ratio as solid-state-laser-based system: a review [1 1240-69]

$112401 \mathrm{U} \quad$ Tomographic imaging with an LED-based photoacoustic-ultrasound system [11240-71]

MICROSCOPY I

$112401 \mathrm{X} \quad$ High-speed label-free galvo mirror scanning-based ultraviolet photoacoustic microscopy for histological imaging [1 1240-74]

FUNCTIONAL, MOLECULAR, AND QUANTITATIVE I

$1124028 \quad$ Ultrafast imaging of cardiac electromechanical wave propagation with volumetric optoacoustic tomography (Seno Medical Best Paper Award) [11240-85]

iv 
FUNCTIONAL, MOLECULAR, AND QUANTITATIVE III

$112402 \mathrm{~L} \quad$ Modelling of uncertainties in ultrasound sensor locations in photoacoustic tomography [11240-91]

$112402 \mathrm{~N} \quad$ Efficient segmentation of multi-modal optoacoustic and ultrasound images using convolutional neural networks [1 1240-93]

1124020 Reflection mode acousto-optic imaging using a 1-D ultrasound array [11240-94]

\section{NOVEL APPROACHES AND APPLICATIONS}

$112402 \mathrm{R} \quad$ Photoacoustic computed tomography guided microrobots for targeted navigation in intestines in vivo (Seno Medical Best Paper Award) [1 1240-97]

$112402 \mathrm{U} \quad$ In vivo super-resolution photoacoustic computed tomography by localization of single dyed droplets [11240-100]

\section{POSTER SESSION}

$112402 Z$ In vivo evaluation of cerebral venous sinus morphology using pulsed-laser-diode-based desktop photoacoustic tomography system [11240-107]

$1124030 \quad$ Photoacoustic imaging of live chicken embryo at multiple developmental stages [11240-108]

1124038 Deep learning-enhanced LED-based photoacoustic imaging [11240-116]

1124039 In vivo visualization of blood vessels in mouse ear by photoacoustic microscopy with transmissive liquid-crystal adaptive optics [1 1240-117]

$112403 \mathrm{H} \quad$ Unsupervised deep learning approach for photoacoustic spectral unmixing [11240-125]

$1124031 \quad$ Design and implementation of low-cost linear laser scanning system for photoacoustic imaging [1 1240-126]

11240 3K Food based contrast agents for photoacoustic imaging [11240-128]

$112403 \mathrm{~N} \quad$ Photoacoustic tomography reconstructing absorption coefficient and effect of regularization minimizing p-norm [11240-132]

11240 3P Vascular and functional imaging by a fast mechanical scanning dual-wavelength photoacoustic microscopy (PAM) [11240-19]

$1124035 \quad$ Photoacoustic imaging using a transvaginal ultrasound probe: a comparison of image reconstruction methods [1 1240-134] 
11240 3V A deep learning method based on U-Net for quantitative photoacoustic imaging [1 1240-137]

$112403 \mathrm{~W} \quad$ Clusters gold nanoparticle-enhanced multimodal photoacoustic microscopy and optical coherence tomography for the identification of choroidal neovascularization in living rabbits [11240-138]

1124032 Improved real-time delay-multiply-and-sum beamforming with coherence factor [11240-141]

1124040 Surface-crosslinked multi-functional nanodroplets for photoacoustic/ultrasound image-guided high intensity focused ultrasound therapy [1 1240-142]

1124043 Monte Carlo simulations and photoacoustic experiments to compare imaging depth at 532 $\mathrm{nm}, 800 \mathrm{~nm}$, and $1064 \mathrm{~nm}$ [11240-145]

$1124044 \quad$ Laser-induced ultrasound transmitters in a flexible photoacoustic and ultrasound tomography setup [11240-146]

11240 4C Multispectral photoacoustic remote sensing microscopy using 532nm and 266nm excitation wavelengths [11240-154]

$112404 G$ Towards quantitative photoacoustic tomography for partial view array transducers [11240-158]

$112404 \mathrm{H} \quad$ Massive parallel ultrasound and photoacoustic PC-based system [1 1240-159]

$112404 \mathrm{~L} \quad$ Deep-tissue photoacoustic imaging at the second near-infrared region using newly synthesized nickel(II) dithioloene-containing polymeric nanoparticles [1 1240-21]

$112404 R$ 3D x-ray induced acoustic computed tomography: a phantom study [1 1240-168]

$112404 \mathrm{U} \quad$ Photoacoustic histology of oxidative stress-induced hippocampal cell death in a mouse brain [11240-171]

$112404 Z \quad$ Evaluation of inflammatory degree using model rats by multi-wavelength photoacoustic imaging system [1 1240-177]

$1124051 \quad$ Real-time improvement of LED-based photoacoustic image quality using intermittent pulse echo acquisitions [11240-179]

1124052 Detecting and evaluating lipid-lich artery plaque using a handheld photoacoustic imaging system [11240-180]

1124054 A GPU approach to real-time coherence-based photoacoustic imaging and its application to photoacoustic visual servoing [11240-182]

1124057 A modular approach to neonatal whole-brain photoacoustic imaging [11240-185]

1124058 Low-cost photoacoustic computed tomography system using light-emitting-diodes [1 1240-186]

1124059 Photoacoustic imaging capabilities of light emitting diodes (LED) and laser sources: a comparison study [11240-187] 
$112405 \mathrm{~A} \quad$ Functional, molecular and structural imaging using LED-based photoacoustic and ultrasound imaging system. [11240-188]

11240 5D Compact photoacoustic add-on for a reflectance confocal microscope [11240-191]

$112405 \mathrm{E} \quad$ A new photoacoustic breast cancer tomography system that images the patient in standing pose [11240-192]

$112405 \mathrm{~F} \quad$ Minimally invasive photoacoustic imaging for device guidance and monitoring of radiofrequency ablation [11240-58] 
Proc. of SPIE Vol. 11240 1124001-8 Downloaded From: https://www.spiedigitallibrary.org/conference-proceedings-of-spie on 25 Apr 2023
Terms of Use: https://www.spiedigitallibrary.org/terms-of-use 


\section{Authors}

Numbers in the index correspond to the last two digits of the seven-digit citation identifier (CID) article numbering system used in Proceedings of SPIE. The first five digits reflect the volume number. Base 36 numbering is employed for the last two digits and indicates the order of articles within the volume. Numbers start with 00, 01, 02, 03, 04, 05, 06, 07, 08, 09, 0A, 0B...0Z, followed by 10-1Z, 20-2Z, etc.

Aaberg, Michael, 3W

Abdelal, Heba, OA

Agano, Toshitaka, $1 T$

Agarwal, Anuradha Murthy, 10

Agrawal, Sumit, 3H, 57, 58, 59, 5A

Albahrani, Hussain, 58, 59, 5A

Alipour, Zahra, OA

Amidi, Eghbal, OA, 4G

Anastasio, Mark A., OL

Anthony, Brian, 10

Ashok, Anuj, 57

Austria Dienzo, Rhonnie, $2 Z$

Awazu, Kunio, $1 \mathrm{~T}$

Baik, Jin Woo, $4 \mathrm{U}$

Bang, Chul Hwan, 06

Bayer, Carolyn L., OD

Bell, Muyinatu A. Lediju, 54

$\mathrm{Bi}$, Renzhe, 3P

Bonaccio, Ermelinda, 5E

Brecht, Hans Peter, $4 \mathrm{H}$

Byun, Kyunghee, $4 \mathrm{U}$

Chapman, William, OA

Chatterjee, Deyali, OA

Chen, Haoyang, 3H, 57

Chen, Tingting, $3 \mathrm{~V}$

Cheng, Kai, 3K

Cheung, Christine, 30

Choi, Changhoon, 1Q

Choi, Hae Young, 4U

Choi, Hak-Jong, 4L

Choi, Hyunsik, 40

Choi, Seongwook, 4R

Choi, Wonseok, 04, 06, 0K, 1Q, 3Z, 40

Collot, Mayeul, 18

Cook, Jason, OL

Dangi, Ajay, 57, 58, 59, 5A

Deán-Ben, Xosé Luís, 17, 28, 2N

Degtyaruk, Oleksiy, 17

DeLuna, Frank, 18

Doctor, Allan, 4G

Dumani, Diego S., OL

Durairaj, Deepit Abhishek, 3H

Emelianov, Stanislav Y., OL

Ermilov, Sergey A., OL, 4H

Fadden, Christopher, 58

Fan, X. Cynthia, 5E

Fernandes, Guilherme S. P., 3S

Fujita, Masanori, 3N

Gambhir, Sanjiv Sam, 04
Gao, Feng, 3V

Gao, Wei, 2R

Gasteau, D., 44

Gonzalez, Eduardo, 54

$\mathrm{Ha}$, Jeonghoon, 04

Hahn, Sei Kwang, 40

Han, Ju Hee, 06

Hashimoto, Nobuyuki, 39

Hattori, Hiroki, OC

Haven, Nathaniel J. M., 4C

Henry, Jessica, 3W

Hirasawa, Takeshi, 3N

Hofmann-Wellenhof, Rainer, 5D

Hooper, Sarah, 04

$\mathrm{Hu}$, Peng, 2R

Huang, Kexin, OA

Huda, Kristie, OD

Ichihashi, Fumiyuki, 38, 51

Ishak, Noreen, 30

Ishihara, Miya, $3 \mathrm{~N}$

Ivanov, Vassili, 4H

Jang, Jinah, IP

Jeon, Seungwan, 0J, $3 Z$

Jeong, Unyong, $1 \mathrm{P}$

Jin, Long, OS

Johnstonbaugh, Kerrick, 3H

Jones, Sydney, 3W

Kalloor Joseph, Francis, OM, 1U, 5F

Kang, Lei, $1 \mathrm{X}$

Karri, Sri Phani Krishna, 3H

Kedarisetti, Pradyumna, 4C

Kim, Chulhong, 04, 06, 0J, 0K, 1P, 1Q, 3K, 3Z, 40,

$4 \mathrm{~L}, 4 \mathrm{R}, 4 \mathrm{U}$

Kim, Hyo Jin, $4 \mathrm{U}$

Kim, Hyung Ham, 1P

Kim, Hyungwoo, 4L

Kim, Jeesu, 04, 06, 0K, 1Q, 3K, 40, 4L

Kim, Jin Young, $4 \mathrm{U}$

Kim, Taeyeong, IP

Kondo, Kengo, OC, 4Z, 52

Kothapalli, Sri-Rajasekhar, 3H, 57, 58, 59, 5A

Kruit, Hindrik, 5F

Kurihara, Makoto, 39

Kushibiki, Toshihiro, $3 \mathrm{~N}$

Lafci, Berkan, $2 \mathrm{~N}$

Lee, Changho, $4 \mathrm{~L}, 4 \mathrm{R}$

Lee, Changyeop, 06, 0K

Lee, Donghyun, 1P, 4R

Lee, Ji Hyun, 06 
Lee, Ki Jong, $3 z$

Lee, Kyung Min, 4L

Leskinen, Jarkko, $2 \mathrm{~L}$

Li, Jiao, 3V

Li, Lei, 2R, $2 U$

Li, Xiufeng, $1 X$

Li, Yanxiu, 3W

Liang, Yizhi, OS

Lim, Dong-Jun, 04

Liu, Bing, 3W

Lu, Tong, 3V

Luo, Hongbo, OA, 4G

Ma, Jun, OS

Mallidi, Srivalleesha, OM, OR

Managuli, Ravi, $3 Z$

Manohar, Srirang, 44, 5F

McMahon, Nicholas, 18

Merćep, Elena, $2 \mathrm{~N}$

Miao, Shichao, 3V

Min, Jung-Joon, 4R

Morscher, Stefan, 2N

Mostafa, Atahar, OA

Mutch, Matthew, OA

Nagel, J. R., 44

Namita, Takeshi, OC, 4Z, 52

Nandy, Sreyankar, OA

Nguyen, Van Phuc, 3W

Notsuka, Yusuke, 39

Nowak, Lukasz J., 20

Nuster, Robert, 5D

Nyayapathi, Nikhila, 5E

Ogawa, Kohei, $4 Z$

Okawa, Shinpei, 3N

Olivo, Malini, 3P

Osman, Mohamed, 57

Özbek, Ali, 28

Özsoy, Çağla, 28

Paltauf, Guenther, 5D

Park, Byullee, 04, 06, 1P, 4L

Park, Eun-Yeoung, 04, 3Z, 4R

Park, Gyeong Sin, 06

Park, Jeongwoo, IP

Paulus, Yannis M., 3W

Pavan, Theo Z., 35

Periyasamy, Vijitha, 43

Pramanik, Manojit, 2Z, 30, 43

Pratap, Rudra, 57

Pu, Yang, 3P

Pulkkinen, Aki, 2L

Qian, Thomas, 3W

Qian, Wei, 3W

Rajendran, Praveenbalaji, $2 Z$

Rascevska, Elina, 5F

Razansky, Daniel, 17, 28, 2N

Restall, Brendon S., 4C

Rhie, Jong Won, 06

Rogers, Stephen, 4G

Roy, Kaustav, 57

Ryu, Seon Young, $4 \mathrm{U}$

Sahlström, Teemu, 2L
Sahu, Samiran, $2 Z$

Salehi, Hassan S., 31

Sampaio, Diego R. T., 35

Sankai, Yoshiyuki, 38, 51

Sato, Naoto, 1T, 38, 51

Schad, John D., 31

Sharma, Arunima, 30, 43

Shiina, Tsuyoshi, OC, 4Z, 52

Shiotani, Kazuma, 52

Singh, Mithun Kuniyil Ajith, OM, OR, 1U, 38, 51, 58, $59,5 \mathrm{~A}$

Singh, Robin, 10

Sivasubramanian, Kathyayini, 38, 3K

Son, Myeongjoo, $4 \mathrm{U}$

Song, Shaoze, $3 \mathrm{~V}$

Srishti, Srishti, 43

Steenbergen, Wiendelt, OM, 1U, 20

Steinberg, Idan, 04

Swee-Hin, Teoh, 30

Takabe, Kazuaki, 5E

Takahashi, Eiji, 39

Tarvainen, Tanja, 2L

Thompson, D., 44

Thompson, Weylan, $\mathrm{OL}, 4 \mathrm{H}$

Tiao, Melinda, 5E

Tick, Jenni, 2L

Uddin, K. M. Shihab, OA, 4G

van de Loo, Fons, OM

van der Laken, Conny, OM

van Hespen, J. C. G., 44

Wang, Lidai, OS

Wang, Lihong $V ., 2 R, 2 U$

Wang, Xueding, 3W

Wible, Christopher, 57

Wong, Terence T. W., IX

Wu, Zhiguang, $2 R$

Xavierselvan, Marvin, OM, OR

Xia, Jun, 5E

Xing, Lei, 38, 3K

Yamakawa, Makoto, 0C, 4Z, 52

Yamaoka, Yoshihisa, 39

Yang, Guang, 0A, 4G

Yang, Xinyi, 58, 59, 5A

Yang, Yiran, $2 R$

Ye, Jing Yong, 18

Yong, Uijung, IP

YU, Anthony, OL

Yun, Misun, 4L

Zemp, Roger J., 4C

Zhang, Huijuan, 5E

Zhang, Pengfei, $2 U$

Zhang, Wei, 3W

Zhang, Yan, IX

Zhou, Shuang, OA

Zhu, Quing, OA, 4G 


\title{
Conference Committee
}

\author{
Symposium Chairs
}

Jennifer K. Barton, The University of Arizona (United States)

Wolfgang Drexler, Medizinische Universität Wien (Austria)

Program Track Chairs

E. Duco Jansen, Vanderbilt University (United States)

Jessica C. Ramella-Roman, Florida International University

(United States)

Conference Chairs

Alexander A. Oraevsky, TomoWave Labs, Inc. (United States)

Lihong V. Wang, Caltech (United States)

Conference Program Committee

Mark A. Anastasio, Washington University in St. Louis (United States)

Paul C. Beard, University College London (United Kingdom)

A. Claude Boccara, Institut Langevin Ondes et Images (France)

Peter Burgholzer, Research Center for Non Destructive Testing GmbH (Austria)

Stanislav Y. Emelianov, Georgia Institute of Technology

(United States)

Rinat O. Esenaliev, The University of Texas Medical Branch (United States)

Martin Frenz, Universität Bern (Switzerland)

Miya Ishihara, National Defense Medical College (Japan)

Chulhong Kim, Pohang University of Science and Technology (Korea, Republic of)

Changhui Li, Peking University (China)

Pai-Chi Li, National Taiwan University (Taiwan)

Andreas Mandelis, University of Toronto (Canada)

Srirang Manohar, Universiteit Twente (Netherlands)

Vasilis Ntziachristos, Helmholtz Zentrum München GmbH (Germany)

Matthew O'Donnell, University of Washington (United States)

Günther Paltauf, Karl-Franzens Universität Graz (Austria)

Wiendelt Steenbergen, Universiteit Twente (Netherlands)

Roger J. Zemp, University of Alberta (Canada)

Vladimir P. Zharov, University of Arkansas for Medical Sciences (United States)

Qifa Zhou, The University of Southern California (United States) 
Quing Zhu, Washington University in St. Louis (United States)

\section{Session Chairs}

$1 \quad$ Clinical Imaging I: In Vivo

Alexander A. Oraevsky, TomoWave Labs, Inc. (United States)

Lihong V. Wang, Caltech (United States)

2 Clinical Imaging II: Ex Vivo

Srirang Manohar, Universiteit Twente (Netherlands)

Rinat O. Esenaliev, The University of Texas Medical Branch (United States)

3 Clinical Imaging III: Ex Vivo

Wiendelt Steenbergen, Universiteit Twente (Netherlands)

Miya Ishihara, National Defense Medical College (Japan)

4 Towards Clinical Imaging

Chulhong Kim, Pohang University of Science and Technology (Korea, Republic of)

Miya Ishihara, National Defense Medical College (Japan)

5 Small-Animal Imaging

Vasilis Ntziachristos, Technische Universität München (Germany)

Daniel Razansky, ETH Zurich (Switzerland)

6 Optical Sensing and Generation of US I

Paul C. Beard, University College London (United Kingdom)

Guenther Paltauf, Karl-Franzens Universität Graz (Austria)

7 Optical Sensing and Generation of US II

Peter Burgholzer, Research Center for Non Destructive Testing GmbH (Austria)

Alexander A. Oraevsky, TomoWave Labs, Inc. (United States)

8 Contrast Agents

Stanislav Y. Emelianov, Georgia Tech Research Institute (United States)

Pai-Chi Li, National Taiwan University (Taiwan)

9 Phantoms and Standardization Activities

Sarah Elizabeth Bohndiek, University of Cambridge (United Kingdom) Seonyeong Park, University of Illinois (United States)

10 Endoscopy and Minimally-Invasive

Qifa Zhou, The University of Southern California (United States)

Lihong V. Wang, Caltech (United States) 
11 Multi-modality Imaging

Quing Zhu, Washington University in St. Lovis (United States)

Albert Claude Boccara, Institut Langevin Ondes et Images (France)

12 Microscopy I

Lihong V. Wang, Caltech (United States)

Changhui Li, Peking University (China)

13 Microscopy II

Paul C. Beard, University College London (United Kingdom)

Roger J. Zemp, University of Alberta (Canada)

14 Functional, Molecular, and Quantitative I

Ben T. Cox, University College London (United Kingdom)

Pai-Chi Li, National Taiwan University (Taiwan)

15 Functional, Molecular, and Quantitative II

Roger J. Zemp, University of Alberta (Canada)

Srirang Manohar, Universiteit Twente (Netherlands)

16 Functional, Molecular, and Quantitative III

Chulhong Kim, Pohang University of Science and Technology (Korea, Republic of)

Vasilis Ntziachristos, Technische Universität München (Germany)

17 Novel Approaches and Applications

Vladimir P. Zharov, University of Arkansas for Medical Sciences (United States)

Matthew O'Donnell, University of Washington (United States) 
Proc. of SPIE Vol. 11240 1124001-14

Downloaded From: https://www.spiedigitallibrary.org/conference-proceedings-of-spie on 25 Apr 2023 Terms of Use: https://www.spiedigitallibrary.org/terms-of-use 